\title{
3D Imaging Algorithm for Down-Looking MIMO Array SAR Based on Bayesian Compressive Sensing
}

\author{
Xiaozhen Ren, ${ }^{1}$ Lina Chen, ${ }^{2}$ and Jing Yang ${ }^{1}$ \\ ${ }^{1}$ College of Information Science and Engineering, Henan University of Technology, Zhengzhou 450001, China \\ ${ }^{2}$ Textile College, Henan Institute of Engineering, Zhengzhou 451191, China \\ Correspondence should be addressed to Xiaozhen Ren; rxz235@163.com
}

Received 21 February 2014; Accepted 17 April 2014; Published 11 May 2014

Academic Editor: Wei Xu

Copyright (C) 2014 Xiaozhen Ren et al. This is an open access article distributed under the Creative Commons Attribution License, which permits unrestricted use, distribution, and reproduction in any medium, provided the original work is properly cited.

\begin{abstract}
Down-looking MIMO array SAR can reconstruct 3D images of the observed area in the inferior of the platform of the SAR and has wide application prospects. In this paper, a new strategy based on Bayesian compressive sensing theory is proposed for down-looking MIMO array SAR imaging, which transforms the cross-track imaging process of down-looking MIMO array SAR into the problem of sparse signal reconstruction from noisy measurements. Due to account for additive noise encountered in the measurement process, high quality image can be achieved. Simulation results indicate that the proposed method can provide better resolution and lower sidelobes compared to the conventional method.
\end{abstract}

\section{Introduction}

Traditional synthetic aperture radar (SAR) is a microwave sensor which can reconstruct two-dimensional (2D) images of the observed area with weather independence and all-day operation capabilities $[1,2]$. However, traditional 2D SAR works in side-looking mode and often meets with shading and lay over effects in urban and mountain areas. Compared with 2D SAR, 3D SAR has distinct advantage in estimation of forest height, 3D digital maps, complex terrain mapping, and so on. Multibaseline SAR tomography is an advanced 3D SAR imaging mode, which forms an additional synthetic aperture in the height direction. Therefore, it has resolving capability along this dimension. Unfortunately, for the current SAR tomography, it is almost impossible to avoid an uneven track distribution in repeat-pass data acquisition, which is just the main reason for the strong ambiguity in height $[3,4]$.

Down-looking array SAR is an innovative imaging mode, which obtains range resolution by pulse compression, azimuth resolution by virtual aperture synthesis with platform movement, and cross-track resolution by a linear array antenna [5-7]. Down-looking array SAR can overcome restrictions of shading and lay over effects in side-looking SAR and also avoid the height ambiguity problem in SAR tomography caused by the uneven track distribution. However, in order to avoid the grating lobe effect in the cross-track direction, a large number of antenna elements are required, which increase the cost and complexity of the equipment. Therefore, multiple-input-multiple-output (MIMO) antenna array is often used in down-looking array SAR, which can reduce the number of real antenna elements largely for a given size of antenna array [8-10]. The common methods used for down-looking MIMO array SAR imaging are usually based on matched filter, which often suffer from low resolution and high sidelobe interference in the images [7, 8]. Moreover, the cross-track resolution is limited by the length of linear array. Hence, high resolution imaging algorithms are desired.

In recent years, Bayesian compressive sensing (BCS) has caused widespread concern, showing significant advantages to sparse signal reconstruction [11]. BCS methods provide certain improvements compared with norm-based CS methods in low noise level, by exploiting the sparseness prior distribution of the image scene. In addition, the Bayesian framework takes into account the additive noise encountered when implementing compressed sampling. Therefore, some BCS based methods for SAR applications have been concerned about recently $[12,13]$. As the $3 \mathrm{D}$ illuminated scene contains only a very small strong scattering centers compared 


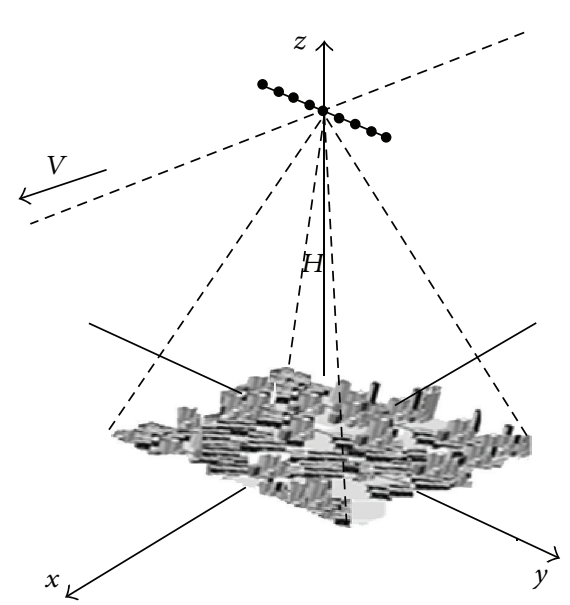

FIGURE 1: Geometry of down-looking MIMO array SAR.

with the total space cells, representing strong spatial sparsity in high frequency radar application, we propose a new 3D imaging algorithm for down-looking MIMO array SAR based on BCS.

The rest of the paper is organized as follows. Section 2 presents the geometry and principle of down-looking MIMO array SAR system. In Section 3, a new 3D imaging algorithm for down-looking MIMO array SAR is described in detail. The performance of the method is investigated in Section 4. Finally, Section 5 gives a brief conclusion.

\section{Down-Looking MIMO Array SAR}

2.1. Geometrical Model. The geometry of down-looking MIMO array SAR is shown in Figure 1. $x, y$, and $r$ denote the azimuth, cross-track, and slant range direction, respectively. The radar platform flies along the $x$-axis corresponding to azimuth direction, with velocity $v$ at height $H$. The thinned linear antenna array, which contains $M$ transmitting antenna elements and $N$ receiving antenna elements, is mounted in the cross-track direction along the wings. The transmitting antenna elements are located at the tips with the distance $d$, the receiving antenna elements are centered at the $y$-axis with spacing $M d / 2$, and the distance between the transmitting and receiving antenna elements is $d / 2$. The thinned linear antenna array works in the time division mode. Each time, only one transmitting antenna element transmits signal and all the receiving antenna elements receive echo simultaneously. The transmitting antenna elements work sequentially and an aperture synthesis period is acquired until all the transmitting antenna elements have worked once. According to the principle of equivalent phase center, the thinned linear array formed by the above positions and work mode is equal to a fully distributed virtual uniform linear array $[5,10]$. The virtual antenna array is composed of $M N$ virtual elements and works in self-transmitting and receiving mode. That is to say, each virtual antenna element transmits and receives signal by itself. These virtual antenna elements are uniform distributed along the wings and centered at the $y$-axis. Each virtual antenna element is located at the mean position of a

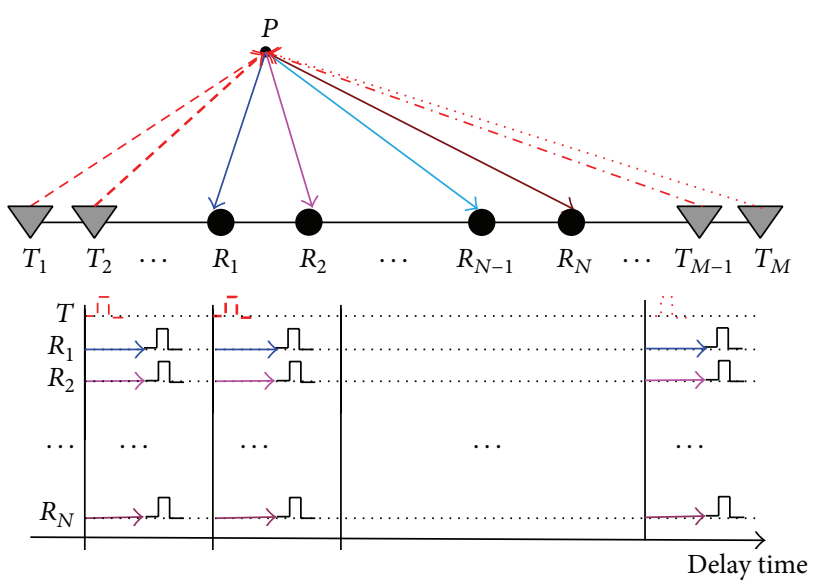

FIGURE 2: Transmitting and receiving order of down-looking MIMO array SAR.

real single transmitting element and a real single receiving element, and the distance between individual virtual antenna elements is $d / 2$. Figure 2 shows the transmitting and the receiving order of each antenna element for a down-looking MIMO array SAR.

2.2. Equivalent Phase Error Compensation. Consider the data acquisition shown in Figure 1 . At the slow time $t_{m}$, the position of the $i$ th transmitting antenna element is given by $\left(x, y_{T i}, H\right)$, where $x=v t_{m}$ is the azimuth position, and $y_{T i}$ is the cross-track position of the $i$ th transmitting antenna element. The position of the $j$ th receiving antenna element is given by $\left(x, y_{R j}, H\right)$, where $y_{R j}$ is the cross-track position of the $j$ th receiving antenna element. For a point scatterer $P$ positioned at $\left(x_{p}, y_{p}, z_{p}\right)$, the transmitting and receiving paths $R_{T i}$ and $R_{R j}$ are given by

$$
\begin{aligned}
R_{T i} & =\sqrt{\left(x-x_{p}\right)^{2}+\left(y_{T i}-y_{p}\right)^{2}+\left(H-z_{p}\right)^{2}} \\
& =\sqrt{\left(y_{T i}-y_{p}\right)^{2}+R_{P}^{2}}, \\
R_{R j} & =\sqrt{\left(x-x_{p}\right)^{2}+\left(y_{R j}-y_{p}\right)^{2}+\left(H-z_{p}\right)^{2}} \\
& =\sqrt{\left(y_{R j}-y_{p}\right)^{2}+R_{p}^{2}},
\end{aligned}
$$

where

$$
R_{p}=\sqrt{\left(x-x_{p}\right)^{2}+\left(H-z_{p}\right)^{2}}
$$

Then the complete travelling path of the wave from the $i$ th transmitting antenna element to point scatterer $P$ to the $j$ th receiving antenna element is given by

$R_{i, j}=R_{T i}+R_{R j}=\sqrt{\left(y_{T i}-y_{p}\right)^{2}+R_{P}^{2}}+\sqrt{\left(y_{R j}-y_{p}\right)^{2}+R_{P}^{2}}$. 
From (2) we can get that the complete travelling path contains two square roots, which will lead to complex computation for the following imaging process. According to the principle of equivalent phase center, the above complete travelling path can be equal to the dual echo paths from the virtual antenna element located at $y=\left(y_{T i}+y_{R j}\right) / 2$ to point scatterer $P$ [5]. And the equivalent echo path can be written as

$$
\begin{aligned}
R & =\sqrt{\left(x-x_{p}\right)^{2}+\left(y-y_{p}\right)^{2}+\left(H-z_{p}\right)^{2}} \\
& =\sqrt{\left(y-y_{p}\right)^{2}+R_{p}^{2}} .
\end{aligned}
$$

Then, the phase difference between the virtual antenna element and the reality antenna element is given by

$$
\begin{aligned}
& \Delta \varphi= \frac{2 \pi}{\lambda}\left(R_{i, j}-2 R\right) \\
&= \frac{2 \pi}{\lambda}\left(\sqrt{\left(y_{T i}-y_{p}\right)^{2}+R_{P}^{2}}+\sqrt{\left(y_{R j}-y_{p}\right)^{2}+R_{P}^{2}}\right. \\
&\left.\quad-2 \sqrt{\left(y-y_{p}\right)^{2}+R_{p}^{2}}\right) \\
& \approx \frac{\pi\left(y_{T i}-y_{R j}\right)^{2}}{2 \lambda R_{p}} .
\end{aligned}
$$

When the scatterers are located at the far field, the phase difference $\Delta \varphi$ is approximate to zero. Otherwise, the phase compensation should be implemented before the image processing. From (5) it can be seen that the compensated phase varies with $R_{p}$; in practice we use the center of view field as reference point to compensate the whole view field.

Furthermore, the down-looking MIMO array SAR works in the time division mode and the virtual antenna elements obtained from different pulse repetition period are not in a straight line with the movement of the platform. Therefore, in order to obtain a fully distributed virtual uniform linear array, the motion compensation should be implemented. The compensated phase caused by the overtake or lag phases owing to the position difference of the antenna elements is given by

$$
\begin{aligned}
& \Delta \varphi_{l, k}=\frac{4 \pi}{\lambda}(\left(\left(\frac{M v}{\mathrm{PRF}}\left(l-\frac{L}{2}\right)+\Delta x_{k}-x_{p}\right)^{2}\right. \\
&+\left.\left(y-y_{p}\right)^{2}+\left(H-z_{p}\right)^{2}\right)^{1 / 2} \\
&-\left(\left(\frac{M v}{\mathrm{PRF}}\left(l-\frac{L}{2}\right)-x_{p}\right)^{2}\right. \\
&\left.\left.+\left(y-y_{p}\right)^{2}+\left(H-z_{p}\right)^{2}\right)^{1 / 2}\right), \\
& l=1,2, \ldots, L ; k=1,2, \ldots, M,
\end{aligned}
$$

where $L$ is the azimuth sample number and $\Delta x_{k}$ is the move spacing between the $k$ th and the first transmitting antenna element in the azimuth direction. $v$ is the velocity of the platform and PRF is the pulse repetition frequency.
After the process above, the collected data of downlooking MIMO array SAR can be regarded as received by the fully distributed virtual linear array.

\section{Three-Dimensional Imaging Algorithm for Down-Looking MIMO Array SAR}

Based on the principle of equivalent phase center, the thinned linear array formed by the time division mode can be equal to a virtual linear array, and each virtual antenna element transmits and receives signal by itself. The linear frequency modulated pulse signal transmitted by the $m$ th virtual antenna element is given by

$$
s(\widehat{t})=\operatorname{rect}\left(\frac{\widehat{t}}{T_{r}}\right) \exp \left[j 2 \pi\left(f_{c} \widehat{t}+\frac{1}{2} \gamma \hat{t}^{2}\right)\right],
$$

where $\hat{t}$ denotes the fast time, $T_{r}$ denotes the pulse width, $f_{c}$ is the carrier frequency, $\gamma$ is the chirp rate, and $\operatorname{rect}(\cdot)$ is the unit rectangular function.

For an arbitrary point scatterer positioned at $P\left(x_{p}, y_{p}, z_{p}\right)$, the echo signal received by the $m$ th virtual antenna element can be expressed as

$$
\begin{aligned}
s_{1}\left(\widehat{t}, \tau, y_{m}\right)= & \operatorname{rect}\left(\frac{\hat{t}-2 R / c}{T_{r}}\right) \operatorname{rect}\left(\frac{\tau-\tau_{p}}{T_{a}}\right) \\
& \times \exp \left[j \pi \gamma\left(\widehat{t}-\frac{2 R}{c}\right)^{2}-j \frac{4 \pi}{\lambda} R\right],
\end{aligned}
$$

where $\tau$ denotes the azimuth time, $\tau_{p}=x_{p} / v$ is the azimuth time of the point scatterer $P, c$ is the light velocity, $T_{a}$ is the observing duration, $\lambda$ is the wavelength, and $R$ is the instantaneous distance between the $m$ th virtual antenna element and the point scatterer

$$
\begin{aligned}
R & =\sqrt{\left(v \tau-x_{p}\right)^{2}+\left(y_{m}-y_{p}\right)^{2}+\left(H-z_{p}\right)^{2}} \\
& \approx R_{p}+\frac{\left(v \tau-x_{p}\right)^{2}}{2 R_{p}}+\frac{y_{m}^{2}-2 y_{m} y_{p}}{2 R_{p}}
\end{aligned}
$$

where $R_{p}=\sqrt{\left(H-z_{p}\right)^{2}+y_{p}^{2}}$ and $y_{m}$ is the cross-track position of the $m$ th virtual antenna element.

3.1. Range Compression. Transform the signal expressed in (8) into the range frequency domain

$$
\begin{aligned}
& S_{1}\left(f_{r}, \tau, y_{m}\right) \\
& =\operatorname{rect}\left(\frac{f_{r}}{T_{r} \gamma}\right) \operatorname{rect}\left(\frac{\tau-\tau_{p}}{T_{a}}\right) \\
& \quad \times \exp \left(-j \frac{\pi f_{r}^{2}}{\gamma}\right) \exp \left(-j \frac{4 \pi f_{r}}{c} R\right) \exp \left(-j \frac{4 \pi}{\lambda} R\right),
\end{aligned}
$$

where $f_{r}$ is range frequency. 
The matched filter function for the range compression is given by

$$
H_{1}\left(f_{r}\right)=\operatorname{rect}\left(\frac{f_{r}}{T_{r} \gamma}\right) \exp \left(j \frac{\pi f_{r}^{2}}{\gamma}\right) .
$$

After the range compression, the received signal can be written as

$$
\begin{aligned}
s_{2}\left(\widehat{t}, \tau, y_{m}\right)= & \operatorname{rect}\left(\frac{\tau-\tau_{p}}{T_{a}}\right) \operatorname{sinc}\left[T_{r} \gamma\left(\widehat{t}-\frac{2 R}{c}\right)\right] \\
& \times \exp \left(-j \frac{4 \pi R}{\lambda}\right) .
\end{aligned}
$$

3.2. Azimuth Compression. From (9) and (12) we get that the distance between the antenna element and the scatterer varies with the azimuth position of the radar platform, which leads to the coupling of the envelope in the range-azimuth plane. Therefore, the range migration in the azimuth direction should be removed first before the azimuth compression. And the correction function for the range migration can be derived from (9)

$$
H_{2}\left(f_{r}, \tau\right)=\exp \left(j \frac{2 \pi\left(v \tau-x_{p}\right)^{2}}{c R_{p}} f_{r}\right) .
$$

Transform the signal expressed in (12) to the range frequency domain and correct the range migration using $H_{2}\left(f_{r}, \tau\right)$. Then, after the range inverse Fourier transform, the signal becomes

$$
\begin{aligned}
s_{3}\left(\widehat{t}, \tau, y_{m}\right)= & \operatorname{rect}\left(\frac{\tau-\tau_{p}}{T_{a}}\right) \operatorname{sinc}\left[T_{r} \gamma\left(\hat{t}-\frac{2 R_{c}}{c}\right)\right] \\
& \times \exp \left(-j \frac{4 \pi R_{p}}{\lambda}\right) \cdot \exp \left[-j \frac{2 \pi v^{2}\left(\tau-\tau_{p}\right)^{2}}{\lambda R_{p}}\right] \\
& \times \exp \left[-j \frac{2 \pi\left(y_{m}^{2}-2 y_{m} y_{p}\right)}{\lambda R_{p}}\right],
\end{aligned}
$$

where $R_{c}=R_{p}+\left(y_{m}^{2}-2 y_{m} y_{p}\right) / 2 R_{p}$.

An azimuth Fourier transform is then performed on each range gate to transform the data into the range time-azimuth frequency domain, and the signal becomes

$$
\begin{aligned}
S_{3}\left(\widehat{t}, f_{a}, y_{m}\right) \\
=\operatorname{rect}\left(\frac{f_{a}}{\gamma_{a} T_{a}}\right) \operatorname{sinc}\left[T_{r} \gamma\left(\widehat{t}-\frac{2 R_{c}}{c}\right)\right] \\
\quad \times \exp \left[-j \frac{2 \pi\left(y_{m}^{2}-2 y_{m} y_{p}\right)}{\lambda R_{p}}\right] \cdot \exp \left(-j \frac{4 \pi R_{p}}{\lambda}\right) \\
\quad \times \exp \left[j \frac{\pi f_{a}^{2}}{\gamma_{a}}\right] \exp \left(-j 2 \pi \tau_{p} f_{a}\right),
\end{aligned}
$$

where $\gamma_{a}=2 v^{2} / \lambda R_{p}$. Then a multiplication of the signal with the azimuth matched filter function $H_{3}\left(f_{a}\right)$ is performed, where

$$
H_{3}\left(f_{a}\right)=\exp \left(-j \frac{\pi f_{a}^{2}}{\gamma_{a}}\right) .
$$

Then, we get the azimuth compressed signal by performing inverse Fourier transform in the azimuth direction

$$
\begin{aligned}
s_{4}\left(\hat{t}, \tau, y_{m}\right)= & \operatorname{sinc}\left[T_{r} \gamma\left(\hat{t}-\frac{2 R_{c}}{c}\right)\right] \operatorname{sinc}\left[T_{a} \gamma_{a}\left(\tau-\tau_{p}\right)\right] \\
& \cdot \exp \left[-j \frac{2 \pi\left(y_{m}^{2}-2 y_{m} y_{p}\right)}{\lambda R_{p}}\right] \exp \left(-j \frac{4 \pi R_{p}}{\lambda}\right) .
\end{aligned}
$$

3.3. Cross-Track Compression. From (17) it can be seen that the distance between the antenna element and the scatterer also varies with the cross-track position of the antenna element. Therefore, the range migration in the cross-track direction should be removed before the cross-track compression. And the amount of the range migration to be corrected can be given by (9)

$$
\Delta R_{c}=\frac{y_{m}^{2}-2 y_{m} y_{p}}{2 R_{p}} .
$$

Transform the signal expressed in (17) into the range frequency domain, and correct the range migration in the range frequency domain. Then the signal becomes

$$
\begin{aligned}
s_{4}\left(\hat{t}, \tau, y_{m}\right) & =\operatorname{sinc}\left[T_{r} \gamma\left(\widehat{t}-\frac{2 R_{p}}{c}\right)\right] \operatorname{sinc}\left[T_{a} \gamma_{a}\left(\tau-\tau_{p}\right)\right] \\
& \cdot \exp \left(-j \frac{2 \pi y_{m}^{2}}{\lambda R_{p}}\right) \exp \left(j \frac{4 \pi y_{m} y_{p}}{\lambda R_{p}}\right) \exp \left(-j \frac{4 \pi R_{p}}{\lambda}\right) .
\end{aligned}
$$

The first phase term in (19) represents a quadratic distortion, which can be compensated by

$$
H_{4}\left(y_{m}\right)=\exp \left(j \frac{2 \pi y_{m}^{2}}{\lambda R_{p}}\right) .
$$

In high frequency radar application, the interest scene can be modeled by a limited number of strong scattering centers reflecting impinging electromagnetic waves isotropically to all receivers, representing strong spatial sparsity [14]. Therefore, the cross-track imaging process of down-looking MIMO array SAR can be transformed into the problem of sparse signal reconstruction from noisy measurements. After the $2 \mathrm{D}$ imaging process in the range and azimuth directions, the signal in the range-azimuth cell corresponding to $(\widehat{t}=$ $2 R_{p} / c, \tau=\tau_{p}$ ) by neglecting the constant phase term of (19) can be written as

$$
s_{5}\left(\hat{t}, \tau, y_{m}\right)=\sum_{p=1}^{P} \sigma_{p}(\widehat{t}, \tau) \exp \left(j 2 \pi f_{p} y_{m}\right),
$$


where $\sigma_{p}(\hat{t}, \tau)$ and $f_{p}=2 y_{p} / \lambda R_{p}$ denote the backward scattering coefficient and the frequency of the pth point scatterer, respectively.

For numerical analysis, (21) can be described by discrete system model

$$
\mathbf{s}=\Phi \sigma
$$

where $\mathbf{s}=\left[s_{5}\left(\hat{t}, \tau, y_{1}\right), s_{5}\left(\widehat{t}, \tau, y_{2}\right), \ldots, s_{5}\left(\hat{t}, \tau, y_{M N}\right)\right]^{T}$ is the signal vector corresponding to the $M N$ virtual antenna elements, $\boldsymbol{\sigma}=\left[\sigma_{1}(\widehat{t}, \tau), \sigma_{2}(\widehat{t}, \tau), \ldots, \sigma_{P}(\widehat{t}, \tau)\right]^{T}$ is the complexvalued scatter coefficient vector in the cross-track direction, and the matrix $\Phi$ can be constructed as

$$
\Phi=\left[\phi_{1}, \phi_{2}, \ldots, \phi_{p}, \ldots, \phi_{P}\right],
$$

where

$$
\begin{aligned}
\boldsymbol{\phi}_{p}= & \exp \left(j 2 \pi f_{p} y_{1}\right), \\
& \left.\exp \left(j 2 \pi f_{p} y_{2}\right), \ldots, \exp \left(j 2 \pi f_{p} y_{M N}\right)\right]^{T},
\end{aligned}
$$

where $(\cdot)^{T}$ represents the transpose operation.

In the more realistic case some noise is added on the measurements

$$
\mathbf{s}=\boldsymbol{\Phi} \boldsymbol{\sigma}+\mathbf{n}
$$

with $\mathbf{n}$ a complex Gaussian vector with zero mean and power $\sigma_{n}^{2}$.

Then, the Bayesian compressive sensing method is employed to estimate the $\boldsymbol{\sigma}$. From the aspect of denoising, Laplace distribution is often used as the sparseness prior [15]. Hence, the probability distribution function of $\sigma$ can be given by

$$
\begin{aligned}
p\left(\boldsymbol{\sigma} \mid \sigma_{\delta}\right) & =\prod_{i=1}^{P} p\left(\sigma_{i} \mid \sigma_{\delta}\right) \\
& =\prod_{i=1}^{P} \frac{1}{\sqrt{2} \sigma_{\delta}} \exp \left(-\frac{\sqrt{2} \sigma_{i}}{\sigma_{\delta}}\right),
\end{aligned}
$$

where $\sigma_{\delta}$ is the scale parameter of the Laplace distribution.

And the probability density function of Gaussian noise $\mathbf{n}$ is given by

$$
p\left(\mathbf{n} \mid \sigma_{n}^{2}\right)=\left(\frac{1}{2 \pi \sigma_{n}^{2}}\right)^{M N} \exp \left(-\frac{\|\mathbf{n}\|_{2}^{2}}{2 \sigma_{n}^{2}}\right),
$$

where $\|\cdot\|_{2}^{2}$ denotes the $l_{2}$ norm.

Therefore, the likelihood function of the received data $\mathbf{s}$ is

$$
p\left(\mathbf{s} \mid \boldsymbol{\sigma}, \sigma_{n}^{2}\right)=\left(\frac{1}{2 \pi \sigma_{n}^{2}}\right)^{M N} \exp \left\{-\frac{\|\mathbf{s}-\boldsymbol{\Phi} \boldsymbol{\sigma}\|_{2}^{2}}{2 \sigma_{n}^{2}}\right\} .
$$

Based on Bayesian theory, the maximum a posteriori (MAP) estimator is used to estimate $\sigma$ as

$$
\begin{aligned}
\widehat{\boldsymbol{\sigma}} & =\arg \max [p(\boldsymbol{\sigma} \mid \mathbf{s})] \\
& =\arg \max \left[p\left(\mathbf{s} \mid \boldsymbol{\sigma}, \sigma_{n}^{2}\right) \cdot p\left(\boldsymbol{\sigma} \mid \sigma_{\delta}\right)\right] .
\end{aligned}
$$

Apparently, the MAP solution of $\sigma$ can be estimated by maximizing the log posterior of $\sigma$ as

$$
\begin{aligned}
\widehat{\boldsymbol{\sigma}} & =\arg \max J(\boldsymbol{\sigma}) \\
& =\arg \max \left[\ln p\left(\mathbf{s} \mid \boldsymbol{\sigma}, \sigma_{n}^{2}\right)+\ln p\left(\boldsymbol{\sigma} \mid \sigma_{\delta}\right)\right] \\
& =\arg \max \left[-\frac{1}{2 \sigma_{n}^{2}}\|\mathbf{s}-\boldsymbol{\Phi} \boldsymbol{\sigma}\|_{2}^{2}-\frac{\sqrt{2}}{\sigma_{\delta}} \sum_{i=1}^{P} \sigma_{i}\right] \\
& =\arg \min \left[\|\mathbf{s}-\boldsymbol{\Phi} \boldsymbol{\sigma}\|_{2}^{2}+\mu\|\boldsymbol{\sigma}\|_{1}\right],
\end{aligned}
$$

where $\mu=2 \sqrt{2} \sigma_{n}^{2} / \sigma_{\delta}$.

Then, the quasi-Newton iterative method with Hessian update scheme is used to solve the optimization problem [16]. The gradient of the objective function $J(\boldsymbol{\sigma})$ with respect to $\sigma$ is given by

$$
\nabla J(\boldsymbol{\sigma})=\widetilde{H}(\boldsymbol{\sigma}) \boldsymbol{\sigma}-2 \boldsymbol{\Phi}^{H} \mathbf{s},
$$

where

$$
\begin{aligned}
& \widetilde{H}(\boldsymbol{\sigma})=2 \boldsymbol{\Phi}^{H} \boldsymbol{\Phi}+\mu \boldsymbol{\Lambda}(\boldsymbol{\sigma}), \\
& \boldsymbol{\Lambda}(\boldsymbol{\sigma})=\operatorname{diag}\left\{\left(\left|(\boldsymbol{\sigma})_{i}\right|^{2}+\varepsilon\right)^{-1 / 2}\right\} .
\end{aligned}
$$

Here $\widetilde{H}(\boldsymbol{\sigma})$ is used as an approximation to the Hessian, and $\sigma$ can be obtained from the following quasi-Newton iteration:

$$
\widehat{\boldsymbol{\sigma}}^{(n+1)}=\widehat{\boldsymbol{\sigma}}^{(n)}-\gamma\left[\widetilde{H}\left(\widehat{\boldsymbol{\sigma}}^{(n)}\right)\right]^{-1} \nabla J\left(\widehat{\boldsymbol{\sigma}}^{(n)}\right),
$$

where $\gamma$ is the step size. After substituting (31) into (33), we obtain the following iterative algorithm:

$$
\widetilde{H}\left(\widehat{\boldsymbol{\sigma}}^{(n)}\right) \widehat{\boldsymbol{\sigma}}^{(n+1)}=(1-\gamma) \widetilde{H}\left(\widehat{\boldsymbol{\sigma}}^{(n)}\right) \widehat{\boldsymbol{\sigma}}^{(n)}+2 \gamma \boldsymbol{\Phi}^{H} \mathbf{s} .
$$

The iteration is stopped when $\left\|\widehat{\boldsymbol{\sigma}}^{(n+1)}-\widehat{\boldsymbol{\sigma}}^{(n)}\right\|_{2}^{2}<\zeta$, where $\zeta$ is a small positive constant.

Then, the 3D image of down-looking MIMO array SAR can be obtained until all the range-azimuth cells have been processed using the same procedure.

In conclusion, the $3 \mathrm{D}$ imaging processing flow of the proposed method for down-looking MIMO array SAR can be shown in Figure 3.

3.4. Estimation of the Parameters $\sigma_{n}^{2}$ and $\sigma_{\delta}$. It is clear that the imaging performance of the proposed method is related to the correct selection of the noise level $\sigma_{n}^{2}$. Therefore, the noise level must be estimated accurately. Because the noise always distributes evenly and there are many range cells containing noise only in down-looking SAR imaging, the noise level estimation is available by setting an energy-based threshold to select the noise cells [17]. The threshold is given by

$$
\text { thres }=E_{m}+\left[\sum_{i=1}^{P Q} \frac{\left(E_{i}-E_{m}\right)^{2}}{P Q}\right]^{1 / 2} \text {, }
$$




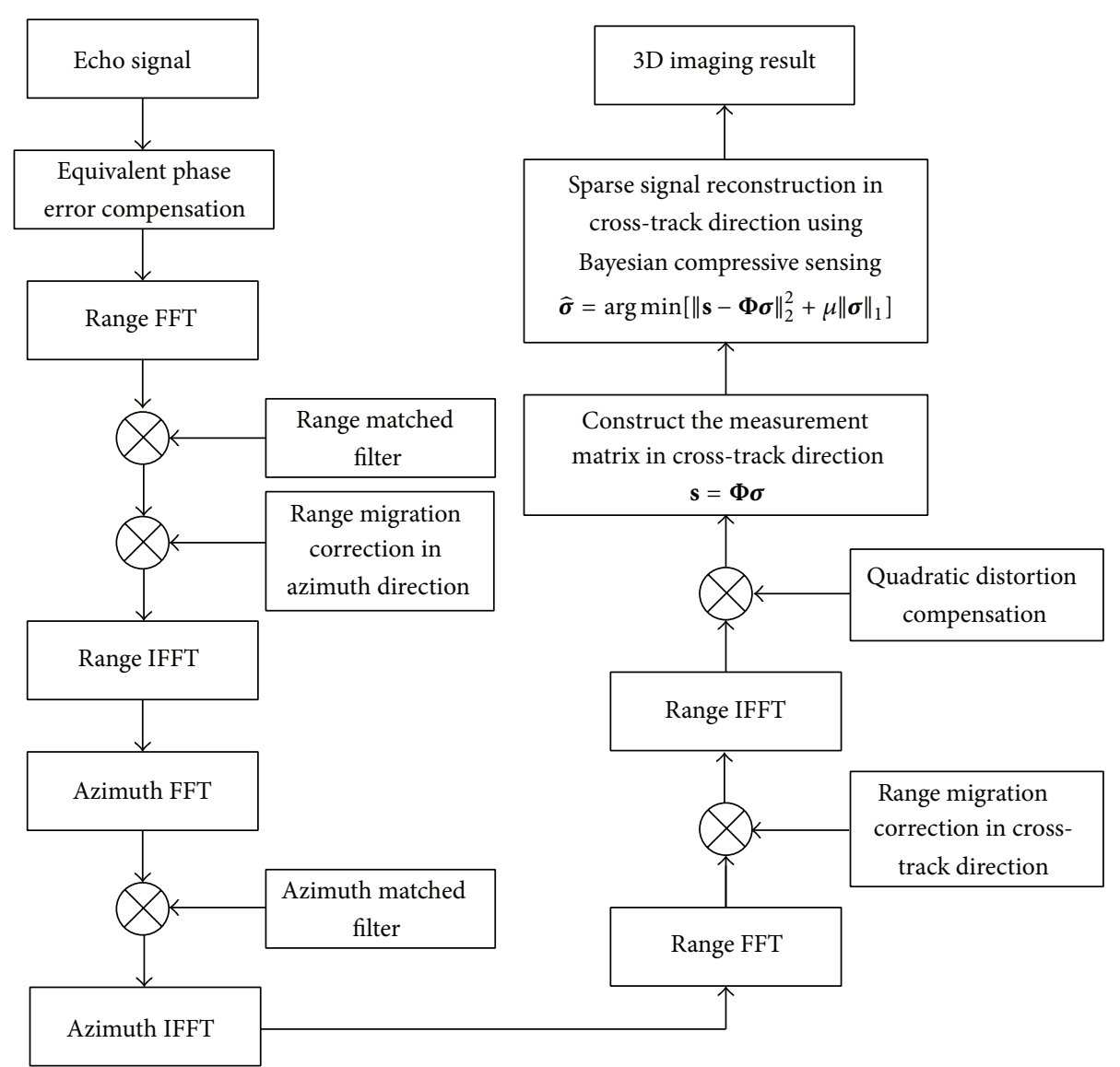

FIGURE 3: The flowchart of proposed method for down-looking MIMO array SAR imaging.

where $E_{i}$ is the energy of the $i$ th range cell and $E_{m}$ denotes the mean energy of all range cells. If the energy of a range cell is below the threshold, this range cell is selected as noise cell. Then, the energy of all the selected cells can be used as the noise level $\sigma_{n}^{2}$.

In addition, the maximum likelihood estimate method is used to choose the objective statistical parameter $\sigma_{\delta}$. According to (26), the log-likelihood function for the Laplace distribution can be written as

$$
\ell\left(\sigma_{\delta} \mid \sigma_{1}, \sigma_{2}, \ldots, \sigma_{P}\right)=-P \ln \sqrt{2} \sigma_{\delta}-\sum_{i=1}^{P} \frac{\sqrt{2} \sigma_{i}}{\sigma_{\delta}}
$$

Maximizing the log likelihood function (36) with respect to $\sigma_{\delta}$ produces the following equation:

$$
\widehat{\sigma}_{\delta}=\frac{1}{P} \sum_{i=1}^{P} \sqrt{2} \sigma_{i} .
$$

From (37) it is clear that we can average the estimates of all pixel values to obtain the estimation of the statistical parameter $\sigma_{\delta}$.
TABLE 1: Parameters used for simulation.

\begin{tabular}{lc}
\hline Parameter & Value \\
\hline Carrier frequency & $37.5 \mathrm{GHz}$ \\
Pulse bandwidth & $300 \mathrm{MHz}$ \\
Pulse repetition frequency & $1024 \mathrm{~Hz}$ \\
Chirp duration & $1.0 \mu \mathrm{s}$ \\
Radar height & $500 \mathrm{~m}$ \\
Radar velocity & $50 \mathrm{~m} / \mathrm{s}$ \\
Number of transmitting antenna elements & 4 \\
Number of receiving antenna elements & 32 \\
Azimuth resolution & $0.4 \mathrm{~m}$ \\
Range resolution & $0.5 \mathrm{~m}$ \\
Cross-track resolution & $0.4 \mathrm{~m}$ \\
\hline
\end{tabular}

\section{Simulation Results}

In this section, point target simulation is carried out to verify the validity of the proposed imaging algorithm. The main parameters used for simulation are listed in Table 1.

Suppose that there are five point targets located at the scene with the azimuth-range-cross track values equal to $(0$, $485,0),(8,495,20),(8,495,-20),(-8,495,20)$, and $(-8$, $495,-20)$, respectively. The distributions of the five point targets are shown in Figure 4(a). After raw data generation 


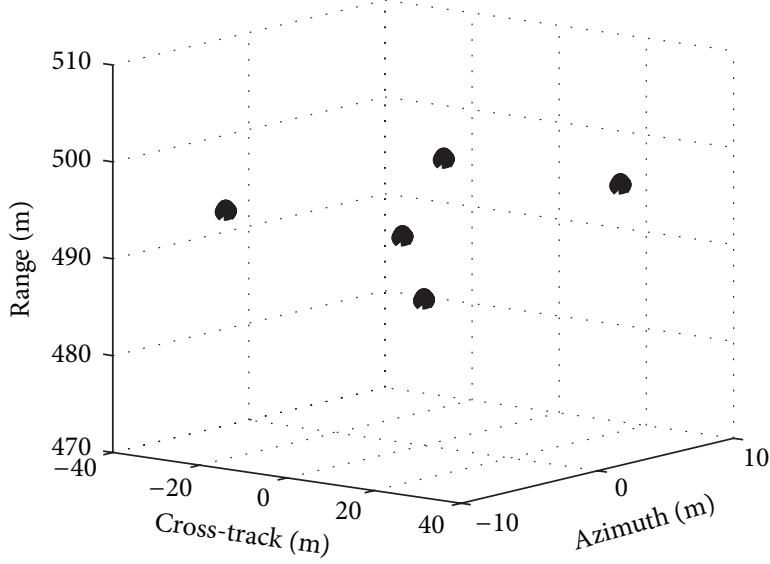

(a) Real spatial position

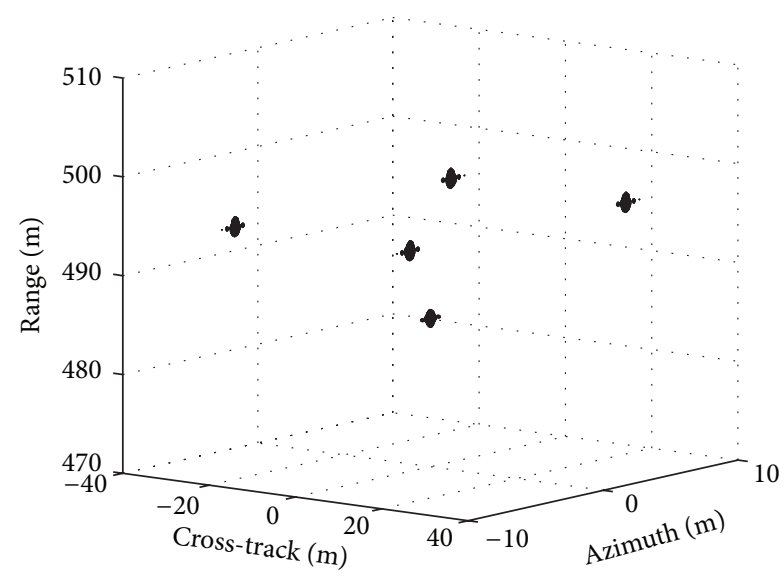

(b) Final 3D image

FIgURE 4: Real spatial position and final 3D image.

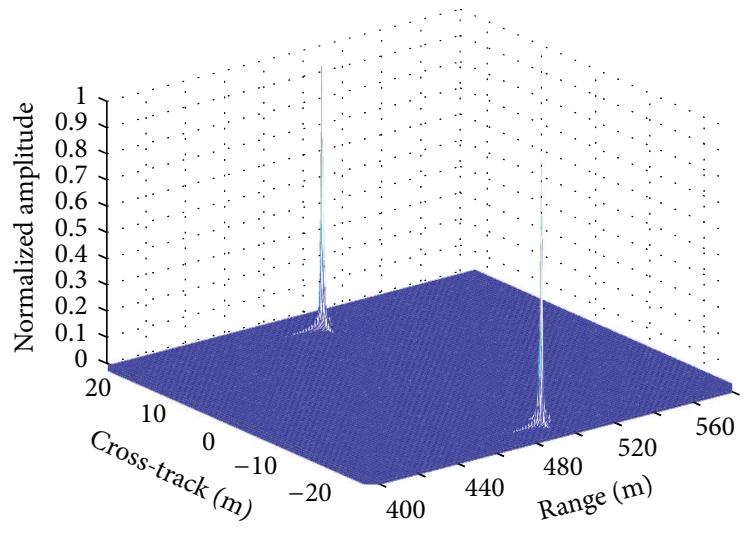

(a) Selected range-cross track section

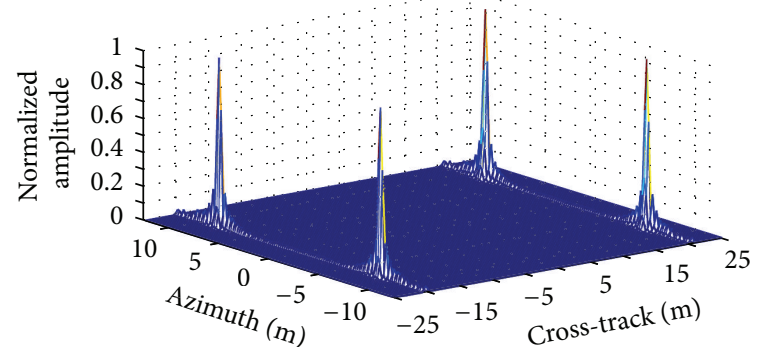

(b) Selected azimuth-cross track section

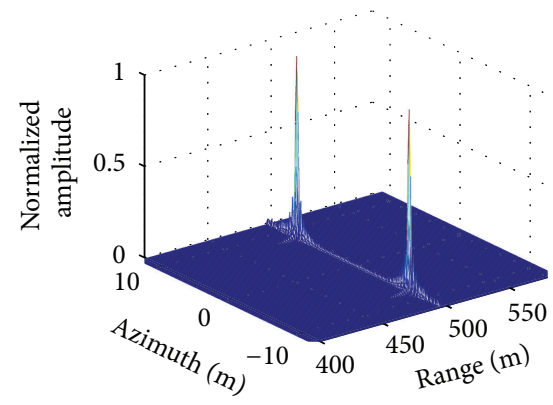

(c) Selected azimuth-range section

FIgURE 5: 2D image of selected sections.

and 3D imaging processing by using the proposed algorithm, the surfaces of the final 3D image are plotted at $-20 \mathrm{~dB}$ in Figure 4(b). As expected, the image is reconstructed in $3 \mathrm{D}$ space, and the whole space structure is very consistent with the real situation in Figure 4(a). Figure 5 shows three selected sections of the final 3D image of down-looking MIMO array SAR. Figure 5(a) shows the 2D image of the selected rangecross track section corresponding to azimuth position $8 \mathrm{~m}$. Figure 5(b) shows the 2D image of the selected azimuthcross track section corresponding to range position $495 \mathrm{~m}$.
Figure 5(c) shows the 2D image of the selected azimuthrange section corresponding to cross-track position $-20 \mathrm{~m}$. The below imaging results show that the point scatterers are well focused in three directions, confirming the validity of the proposed algorithm.

In order to analyze the performance of the proposed method, the imaging result obtained by Fourier transform is given for comparison. Supposed that there are two targets located at the azimuth-range-cross track unit of $(8,0.18,495)$ and $(8,-0.18,495)$. Figure $6($ a) shows the range-cross track 


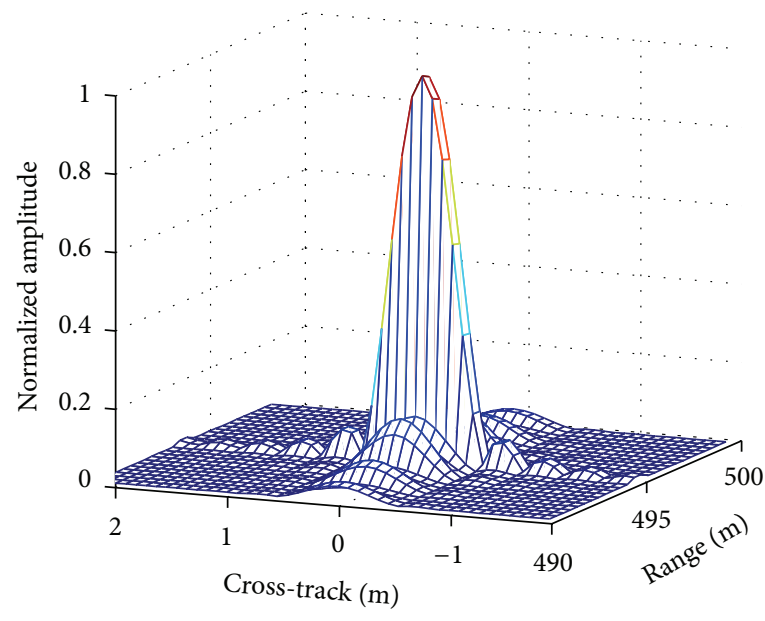

(a) Imaging result obtained by Fourier transform

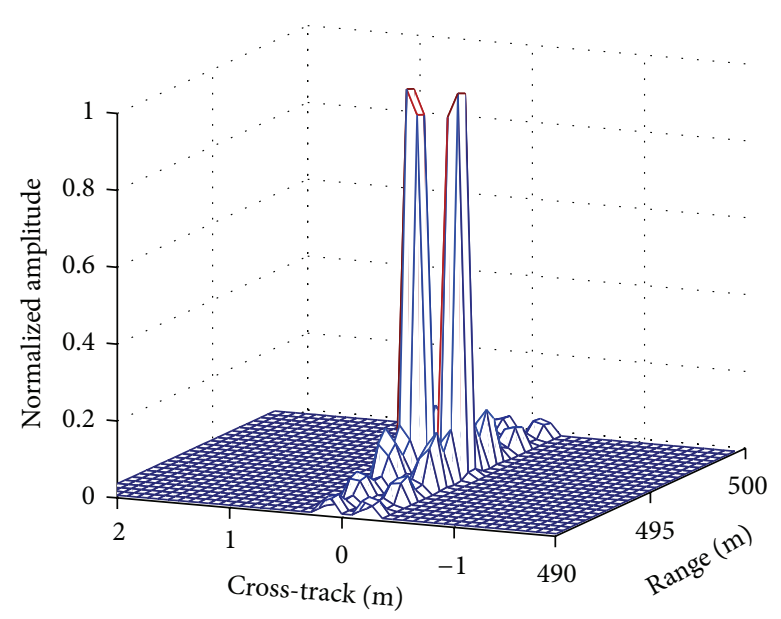

(b) Imaging result obtained by proposed method

FIGURE 6: Comparison of the range-cross track imaging results of down-looking MIMO array SAR.

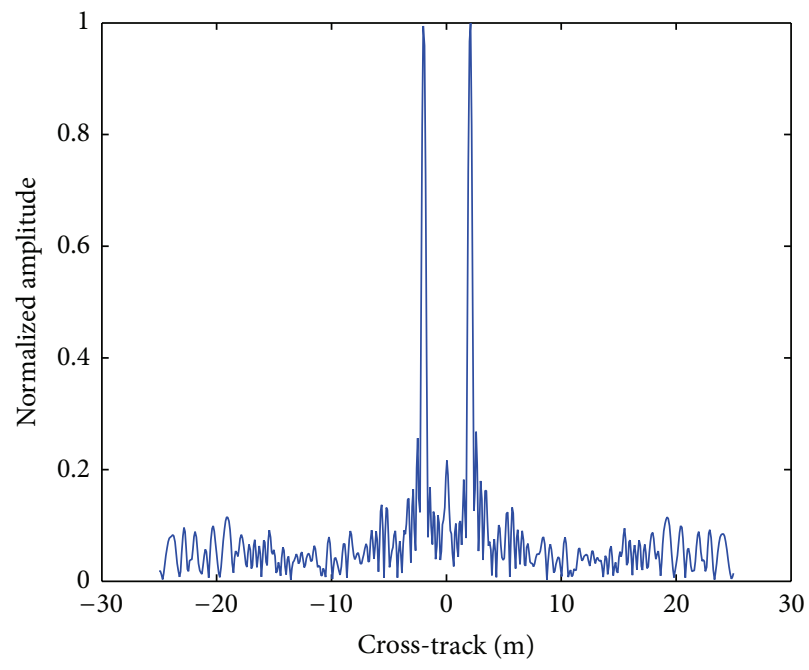

(a) Cross-track distribution obtained by Fourier transform

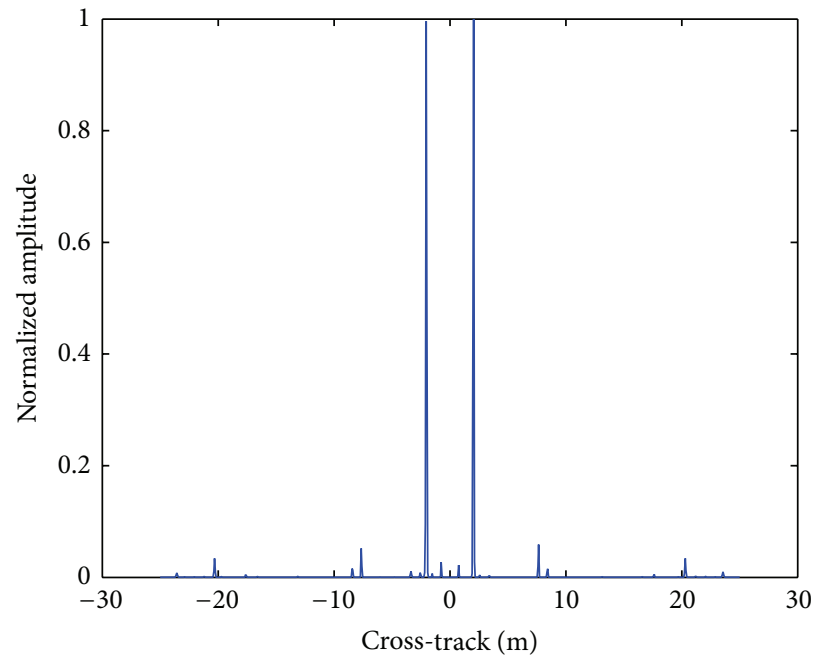

(b) Cross-track distribution obtained by proposed method

Figure 7: Comparison of the cross-track distribution obtained by Fourier transform and the proposed method.

reconstruction result obtained by Fourier transform, and Figure 6(b) shows the range-cross track reconstruction result obtained by the proposed method. As the cross-track distance of the two targets is $0.36 \mathrm{~m}$, which is less than the cross-track resolution of $0.4 \mathrm{~m}$, the two targets can not be distinguished in the image obtained by Fourier based method. However, the proposed method can improve the spatial resolution and distinguish the two targets clearly.

In the following experiment, we take into account two targets located at the same azimuth and range positions and with the cross-track values equal to $-2 \mathrm{~m}$ and $2 \mathrm{~m}$, respectively. Moreover, additional Gaussian distributed complex noise is added to generate measurements with SNR of $5 \mathrm{~dB}$. Figures 7(a) and 7(b) show the cross-track distribution of the two targets obtained by Fourier transform and the proposed method, respectively. By comparing the imaging results, it can be seen that the proposed method is more robust to noise and it recovers target image together with suppressing the noise components.

\section{Conclusions}

Down-looking MIMO array SAR can reconstruct 3D images of the observed area and overcome restrictions of shading and lay over effects in side-looking SAR. Therefore, downlooking MIMO array SAR has challenging potential for 3D digital maps, complex terrain mapping, and so on. However, the cross-track resolution of down-looking MIMO array SAR is limited by the length of linear array. In this paper, a novel 3D imaging strategy is proposed for down-looking MIMO array SAR. Exploiting the spatial sparsity of the interest scene, we transform the cross-track imaging process of downlooking MIMO array SAR into the problem of sparse signal 
reconstruction from noisy measurements. Raw data of downlooking MIMO array SAR in Ka-band is simulated and the 3D image is achieved. The results of the simulated data confirm the effectiveness of the proposed method.

\section{Conflict of Interests}

The authors declare that there is no conflict of interests regarding the publication of this paper.

\section{Acknowledgments}

This work was supported by the National Natural Science Foundation of China under Grant 61201390, Grant 61201389 and Grant 11201120, and the Natural Science Foundation for Education Department of Henan Province under Grant 13 A510184.

\section{References}

[1] D. L. Mensa, High Resolution Radar Imaging, Artech House, Dedham, Mass, USA, 1981.

[2] W. G. Carrara, R. S. Goodman, and R. M. Majewski, Spotlight Synthetic Aperture Radar: Signal Processing and Algorithms, Artech House, Boston, Mass, USA, 1995.

[3] X. Ren, J. Sun, and R. Yang, "A new three-dimensional imaging algorithm for airborne forward-looking SAR," IEEE Geoscience and Remote Sensing Letters, vol. 8, no. 1, pp. 153-157, 2011.

[4] B. Pang, D. Dai, Y. Z. Li et al., "Research on forward-looking synthetic aperture radar imaging algorithm of high velocity platform," in Proceedings of the IET International Radar Conference, 2013.

[5] J. Klare, "A new airborne radar for 3D imaging simulation study of ARTINO," in Proceedings of the 6th European Conference on Synthetic Aperture Radar (EUSAR '06), Dresden, Germany, May 2006.

[6] Y. Wang, L. Du, W. Hong, W. Tan, and Y. Wu, "A threedimensional range migration algorithm for downward-looking 3D-SAR with single-transmitting and multiple-receiving linear array antennas," EURASIP Journal on Advances in Signal Processing, vol. 2010, Article ID 957916, 2010.

[7] X. M. Peng, Y. P. Wang, W. X. Tan et al., "Fast wavenumber domain imaging algorithm for airborne downward-looking array 3d-SAR based on region of interest pick," Journal of Electronics \& Information Technology, vol. 35, no. 7, pp. 15251531, 2013.

[8] J. Klare, "Digital beamforming for a 3D mimo sarimprovements through frequency andwaveform diversity," in Proceedings of the IEEE International Geoscience and Remote Sensing Symposium ((IGARSS '08)), pp. V17-V20, Boston, Mass, USA, July 2008.

[9] W. Q. Wang, "MIMO SAR imaging: potential and challenges," IEEE Aerospace and Electronic Systems Magazine, vol. 28, no. 8, pp. 18-23, 2013.

[10] Y. N. Hou, Study of radar imaging technology based on sparse array antenna [Ph.D. thesis], Institute of Electronics, Chinese Academy of Sciences, 2010.

[11] S. Ji, Y. Xue, and L. Carin, "Bayesian compressive sensing," IEEE Transactions on Signal Processing, vol. 56, no. 6, pp. 2346-2356, 2008.
[12] X. Z. Zhang, J. H. Qin, and G. J. Li, "SAR target classification using bayesian compressive sensing with scattering centers features," Progress in Electromagnetics Research, vol. 136, pp. 385-407, 2013.

[13] J. Xu, Y. Pi, and Z. Cao, "Bayesian compressive sensing in synthetic aperture radar imaging," IET Radar, Sonar \& Navigation, vol. 6, no. 1, pp. 2-8, 2012.

[14] M. A. Herman and T. Strohmer, "High-resolution radar via compressed sensing," IEEE Transactions on Signal Processing, vol. 57, no. 6, pp. 2275-2284, 2009.

[15] S. D. Babacan, R. Molina, and A. K. Katsaggelos, "Bayesian compressive sensing using laplace priors," IEEE Transactions on Image Processing, vol. 19, no. 1, pp. 53-63, 2010.

[16] M. Çetin and W. C. Karl, "Feature-enhanced synthetic aperture radar image formation based on nonquadratic regularization," IEEE Transactions on Image Processing, vol. 10, no. 4, pp. 623631, 2001.

[17] L. Zhang, Z.-J. Qiao, M. Xing, Y. Li, and Z. Bao, "Highresolution ISAR imaging with sparse stepped-frequency waveforms," IEEE Transactions on Geoscience and Remote Sensing, vol. 49, no. 11, pp. 4630-4651, 2011. 

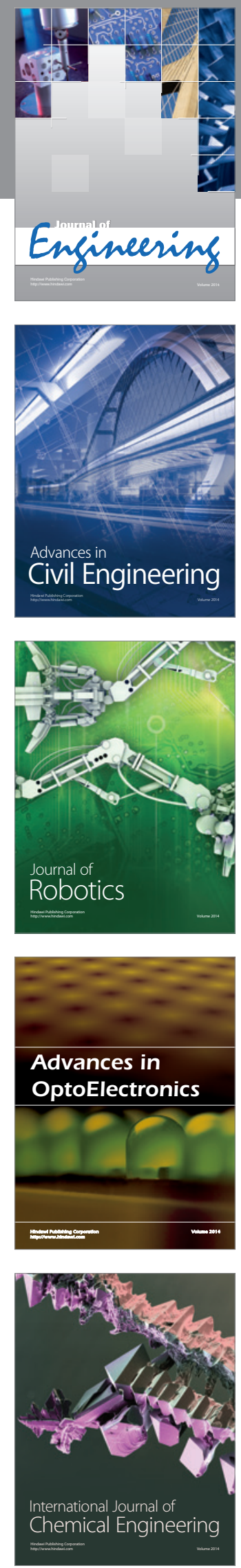

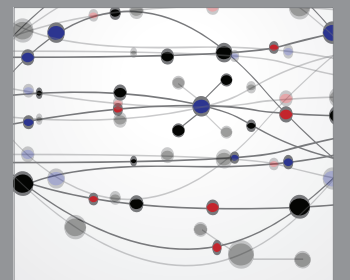

The Scientific World Journal
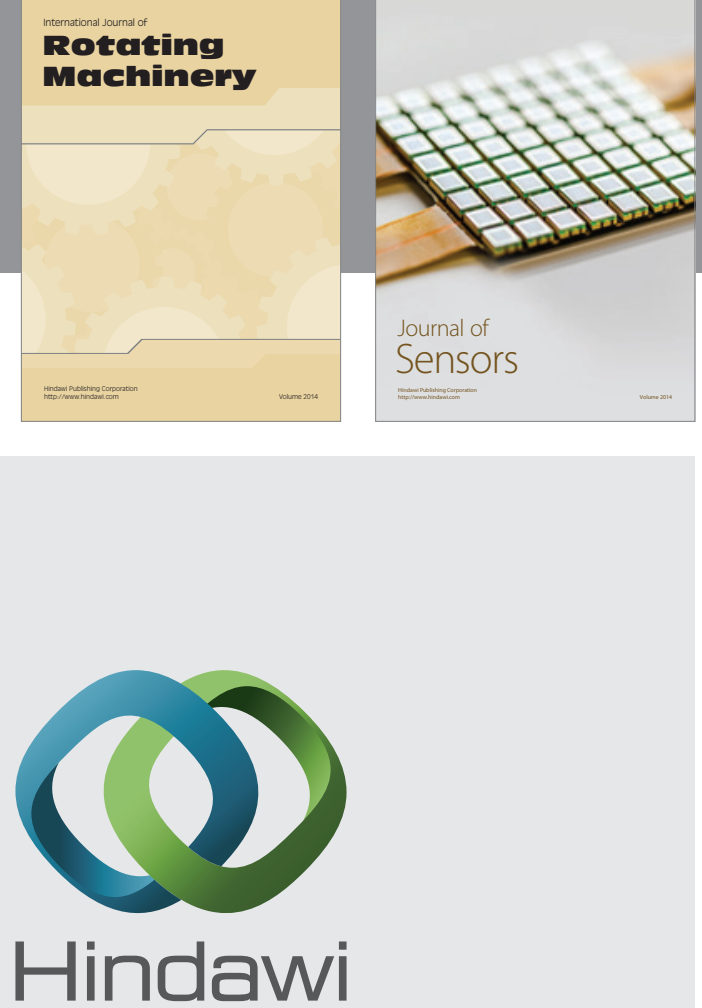

Submit your manuscripts at http://www.hindawi.com
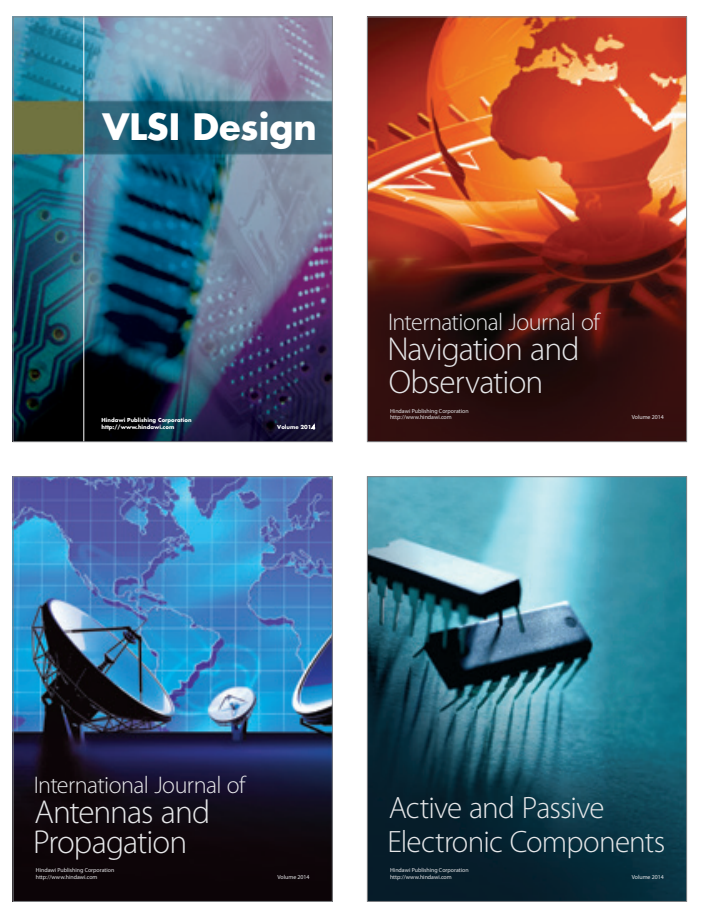
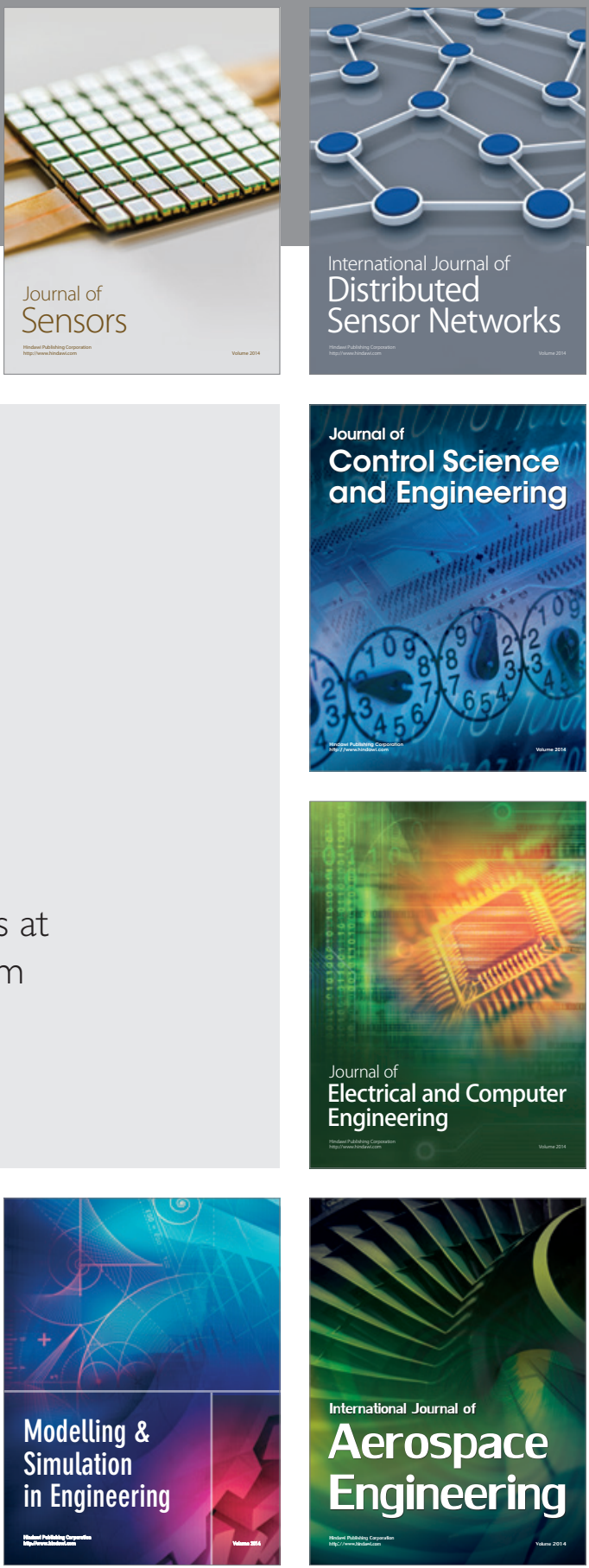

Journal of

Control Science

and Engineering
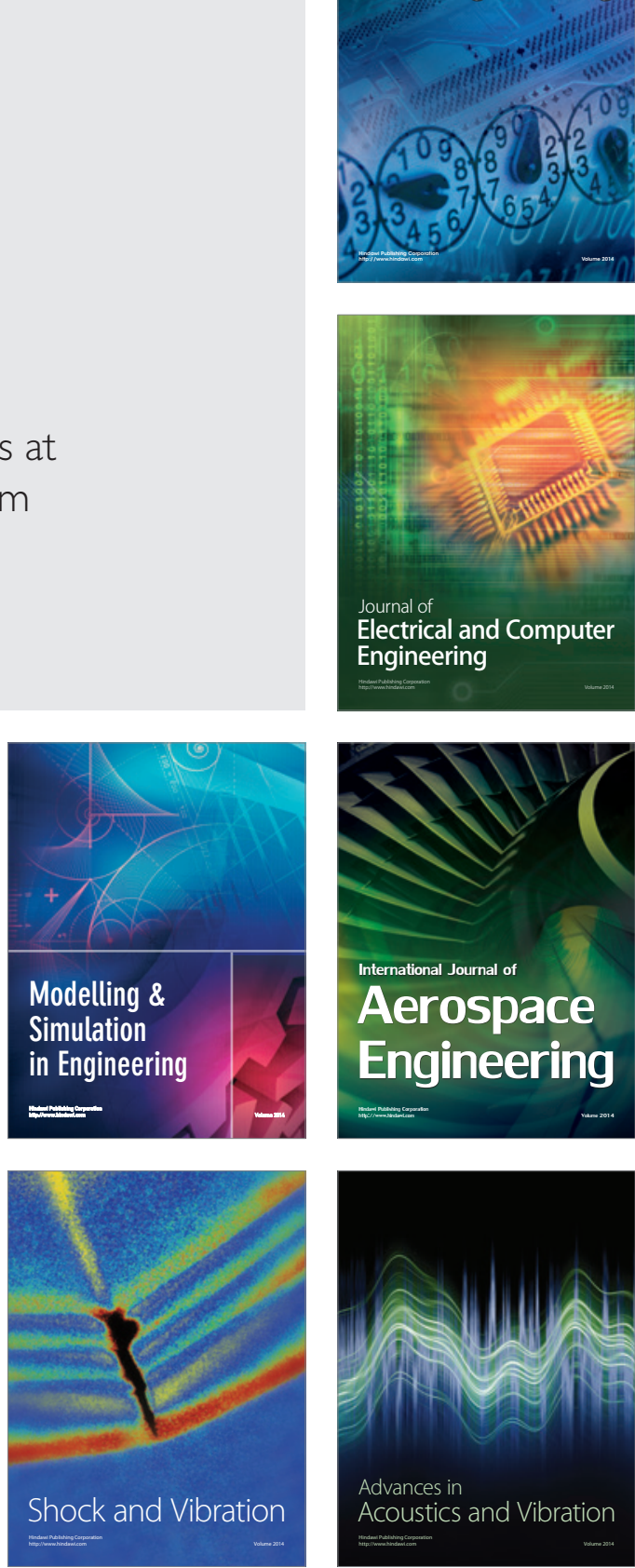